\title{
Uniqueness in chess studies
}

Article

Published Version

Haworth, G. M., Bleicher, E. and van der Heijden, H. M. J. F. (2011) Uniqueness in chess studies. ICGA Journal, 34 (1). pp. 22-24. ISSN 1389-6911 Available at https://centaur.reading.ac.uk/19484/

It is advisable to refer to the publisher's version if you intend to cite from the work. See Guidance on citing.

Publisher: The International Computer Games Association

All outputs in CentAUR are protected by Intellectual Property Rights law, including copyright law. Copyright and IPR is retained by the creators or other copyright holders. Terms and conditions for use of this material are defined in the End User Agreement.

\section{www.reading.ac.uk/centaur}

\section{CentAUR}

Central Archive at the University of Reading

Reading's research outputs online 


\title{
NOTES
}

\section{UNIQUENESS IN CHESS STUDIES}

\author{
G.M C. Haworth, ${ }^{l}$ E. Bleicher and H.M.J.F. van der Heijden
}

Reading, U.K.; Berlin, Germany and Deventer, The Netherlands

\begin{abstract}
Van der Heijden's EndGame StUdy Database IV, HhdbIV, is the definitive collection of 76,132 chess studies. In each one, White is to achieve the stipulated goal, win or draw: study solutions should be essentially unique with minor alternatives at most. In this second note on the mining of the database, we use the definitive Nalimov endgame tables to benchmark White's moves in sub-7-man chess against this standard of uniqueness. Amongst goalcompatible mainline positions and goal-achieving moves, we identify the occurrence of absolutely unique moves and analyse the frequency and lengths of absolutely-unique-move sequences, AUMSs. We identify the occurrence of equi-optimal moves and suboptimal moves and refer to a defined method for classifying their significance.
\end{abstract}

\section{INTRODUCTION}

In a Chess Study, White is required to achieve a stipulated goal, win or draw: the solution should be essentially unique, if not absolutely unique as in a crossword or a Sudoku puzzle. Variants of the solution are duals and range from ignorable through shades of significance to fatal. Sub-7-man (s7m) errors of stipulation or mainline solution in HhdbIV were reviewed in our first note (Bleicher et al., 2010). Ignoring positions and moves failing to achieve the goal, this note reviews the moves from $\mathrm{s} 7 \mathrm{~m}$ wtm mainline positions where the Nalimov Depth To Mate endgame tables, DTM EGTs, provide the definitive truth (Nalimov et al., 2000).

In such positions, two key questions arise:

a) is there a unique optimal move (UOM) or a choice of equi-optimal moves (EOMs), and

b) in Win Studies, are DTM-suboptimal moves also available?

These two questions define a two-dimensional partition of the positions into four sets, see Figure 1:

1) AUM: there is an absolutely unique successful move,

2) $\mathrm{UOM}^{+}$: there is exactly one DTM-optimal and at least one DTM-suboptimal move,

3) OEOM: there are at least two DTM-equi-optimal moves and no others, and

4) $\mathrm{EOM}^{+}$: there are at least two DTM-equi-optimal moves and at least one DTM-suboptimal move.

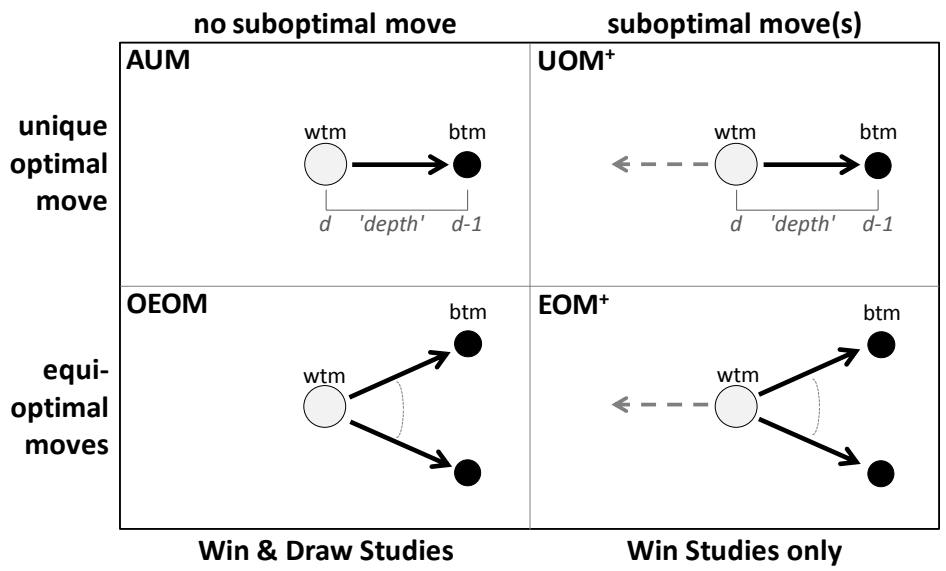

Figure 1. Four sets of s7m wtm positions, partitioned by availability of optimal and DTM-suboptimal moves.

\footnotetext{
${ }^{1}$ The University of Reading, Berkshire, UK, RG6 6AH. email: guy.haworth@bnc.oxon.org.
} 


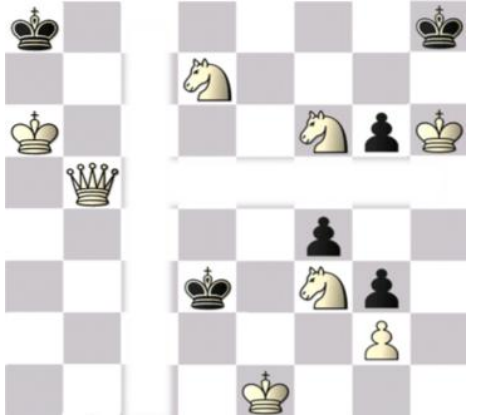

a) Three positions with alternative optimal moves or TWMs

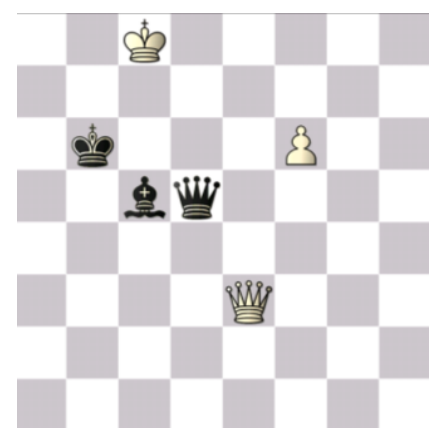

b) db\#49882 8w: J. Vandiest (1981) Sp.p., De Feijter JT, EG

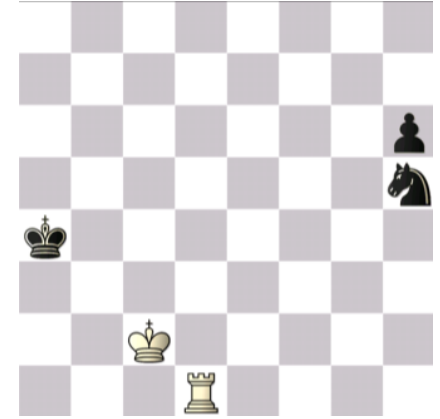

c) db\#70232 1w: H. Conrady (2003)

Computerschach und Spiele

Figure 2. Illustrative positions and longest s7m drawing/winning AUMSs

Absolutely unique moves are the most satisfying aesthetically but essentially unique moves come more than an honourable second as they meet the technical requirements of studies. When suboptimal moves are available, the question is whether they are merely time-wasting moves (TWMs) or not, e.g., moves allowing Black to force a goal-oriented White back to a previous position. If so, they may be considered unambiguously inferior to a move which makes progress towards the study's goal. In Figure 2a (bottom right), at position 8w (White's $8^{\text {th }}$ move) of the 1924 Réti-Mandler KNPKPP study, the moves $\mathrm{Kd} 1$ and $\mathrm{Ne} 5 / \mathrm{Ng} 1 / \mathrm{Ng} 5 / \mathrm{Nh} 4$ are DTMsuboptimal and therefore TWM candidates. Their status as TWMs is easily determined. ${ }^{2}$

More subtly, if there are non-TWM options into sidelines, one might ask whether these are significantly different from the mainline. In Figure 2a, are 1.Qb7\# and 1.Qe8\# significantly different? It is a matter of taste but with an extension of chess notation, there would at least be only one line to write: 1.(Qb7/Qe8)\#. Can other lines be forced to converge quickly with the mainline by a Black wishing to do so? One might argue that the sooner they converge, the less significant they are. Still in Figure $2 \mathrm{a}$, are the lines 1.Nf8 g5 2.Ng6\# and 1.Ne5 g5 2.(Nf7/Ng6)\# significantly different? Again, extending chess notation with [...] meaning any available move, one line suffices: 1.(Ne5/Nf8) g5 2.[Nf7/Ng6]\#.

\begin{tabular}{|c|c|c|c|c|c|}
\hline Statistic & AUM & $\mathbf{U O M}^{+}$ & OEOM & $\mathbf{E O M}^{+}$ & Total \\
\hline s7m wtm positions compatible with the goal & 150,649 & 59,409 & 13,186 & 11,390 & 234,634 \\
\hline$\%$ of all such positions & $64.2 \%$ & $25.3 \%$ & $5.6 \%$ & $4.9 \%$ & $100.0 \%$ \\
\hline s7m wtm positions in Win Studies & 83,978 & 59,409 & 3,939 & 11,390 & 158,716 \\
\hline$\%$ of all such positions & $52.9 \%$ & $37.4 \%$ & $2.5 \%$ & $7.2 \%$ & $100.0 \%$ \\
\hline $\mathrm{s} 7 \mathrm{~m}$ wtm positions in Draw Studies & 66,671 & & 9,247 & & 75,918 \\
\hline$\%$ of all such positions & $87.8 \%$ & & $12.2 \%$ & & $100.0 \%$ \\
\hline \# of 'sideline' DTM-equi-optimal moves & - - & & 26,718 & 17,509 & 44,227 \\
\hline in Win Studies & - & & 5,071 & 17,509 & 22,580 \\
\hline in Draw Studies & & & 21,647 & 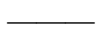 & 21,647 \\
\hline \# of DTM-suboptimal moves (win studies only) & - & 255,344 & & 65,235 & 320,579 \\
\hline \begin{tabular}{|l} 
DTM-suboptimal move played \\
\end{tabular} & $=$ & 8,167 & 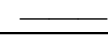 & 1,665 & 9,832 \\
\hline
\end{tabular}

Table 1. A statistical profile of the four sets of positions.

\begin{tabular}{|cccc|ccccc|cccc|ccc|}
\hline Length Wins & Draws & Total & \multicolumn{2}{c|}{ Length Wins } & Draws Total & \multicolumn{2}{c|}{ Length Wins } & Draws Total & \multicolumn{2}{c|}{ Length Wins Draws Total } \\
Total & 34,852 & 17,986 & 52,838 & 8 & 316 & 540 & 856 & 16 & 0 & 33 & 33 & 25 & 0 & 1 & 1 \\
1 & 14,787 & 4,479 & 19,266 & 9 & 177 & 370 & 547 & 17 & 0 & 14 & 14 & 26 & 0 & 1 & 1 \\
2 & 7,752 & 3,207 & 10,959 & 10 & 90 & 270 & 360 & 18 & 1 & 12 & 13 & 33 & 0 & 1 & 1 \\
3 & 4,871 & 2,668 & 7,539 & 11 & 31 & 162 & 193 & 19 & 0 & 11 & 11 & 34 & 0 & 1 & 1 \\
4 & 3,137 & 2,204 & 5,341 & 12 & 17 & 109 & 126 & 20 & 1 & 6 & 7 & 35 & 0 & 1 & 1 \\
5 & 1,911 & 1,688 & 3,599 & 13 & 11 & 73 & 84 & 21 & 0 & 4 & 4 & 36 & 0 & 1 & 1 \\
6 & 1,124 & 1,217 & 2,341 & 14 & 6 & 45 & 51 & 22 & 0 & 9 & 9 & 37 & 0 & 1 \\
7 & 614 & 816 & 1,430 & 15 & 5 & 40 & 45 & 23 & 1 & 1 & 2 & 43 & 0 & 1 \\
\hline
\end{tabular}

Table 2. Counts of Absolutely-Unique-Move Sequences of various lengths.

\footnotetext{
${ }^{2}$ (Beasley, 2003), HHdbIV\#9686. 8.Kd1 Ke3 forces 9.Ke1. Optimal progress is 8.Kf1 Ke3 9.Ne1 Kd2 10.Nc2 Kd3 11.Ke1. Good chess engines quickly dismiss alternative Knight moves as leading to repetition of position.
} 


\section{A STATISTICAL PROFILE OF UNIQUENESS}

Table 1 provides some statistics about both the positions in the four position-sets defined above and the moves from those positions.

Some $24,576 \mathrm{wtm}$ positions offer a total of 44,227 DTM-equi-optimal moves off the authors' mainlines, and $70,799 \mathrm{wtm}$ positions offer a total of 320,579 DTM-suboptimal moves into sidelines. These moves may be further analysed by an as yet unimplemented algorithm (Haworth, 2009) to see if the equi-optimals are essentially equivalent to the mainline move, and if the suboptimal moves are merely TWMs. The total of 364,806 moves is not particularly formidable: many can be classified in seconds: ${ }^{3}$ we look forward to a production attack on this challenge. The algorithm defines appropriate chess variants $\operatorname{Chess}(P)$ to define the impact of deeming the positions in set $P$ to be worth a $1 / 2$-point less to White than they actually are.

In 9,832 Win Study positions, White plays a goal-preserving but DTM-suboptimal move which may nevertheless be the best move. ${ }^{4}$ Most but not all of these studies must be considered unsound because of the DTMoptimal alternative move. ${ }^{5}$ Many of these cooks had not been found before HHdbIV.

Table 2 gives the number of AUM-sequences of various lengths in Win and Draw Studies. Perhaps not surprisingly, the Draw Studies' longest AUMSs ${ }^{6}$ are longer than the Win Studies' AUMSs ${ }^{7}$, and there are at least as many drawing AUMSs as winning AUMSs for all lengths greater than five. All have an awesome fascination and one has to wonder at their composers' ingenuity. Finding these tightrope lines in EGTs (Bleicher, 2011) is a challenging task and clearly, many did not even have such a source.

The longest AUM sequence in a draw study is in Vandiest (1981), HHdbIV\#49882, q.v. Figure 2b. KQPKQB, 2K5/8/1k3P2/2bq4/8/4Q3/8/8 w:

8.Qe1 Qa8+ 9.Kd7 ${ }^{\circ}$ \{only move $\}$ Qc6+ 10.Kd8 ${ }^{\circ}$ Qd6+ 11.Ke8 Qf8+ 12.Kd7 ${ }^{\circ}$ Qf7+ 13.Kd8 Qf8+ 14.Kd7 Qd6+ 15.Ke8 Qd5 16.Qh4 Qe6+ 17.Kd8 Qe5 18.Kd7 Qd5+ 19.Ke8 Qe6+ 20.Kd8 ${ }^{\circ}$ Qc6 21.Qe1 Qa8+ 22.Kd7 ${ }^{\circ}$ Qc6+ 23.Kd8 ${ }^{\circ}$ Qd6+ 24.Ke8 Qf8+ 25.Kd7 ${ }^{\circ}$ Qf7+ 26.Kd8 Qf8+ 27.Kd7 Qd6+ 28.Ke8 Qd5 29.Qh4 Qg8+ 30.Kd7 ${ }^{\circ}$ Qf7+ 31.Kd8 Qe6 32.Qh7 Qd6+ 33.Ke8 Qf8+ 34.Kd7 ${ }^{\circ}$ Qd6+ 35.Ke8 Qe6+ 36.Kd8 Qxf6+ 37.Ke8 Qf8+ 38.Kd7 Qd6+ 39.Ke8 Qe5+ 40.Kf7 Qe7+ 41.Kg6 Qe4+ 42.Kg7 Bd4+ 43.Kh6 Qh4+ 44.Kg6 ${ }^{\circ} \mathrm{Qe} 4+$ 45.Kh6 Be3+ 46.Kg7 Bd4+ 47.Kh6 Qh4+ 48.Kg6 ${ }^{\circ} \mathrm{Qg} 4+49 \cdot \mathrm{Kf} 7 \mathrm{Qd} 7+50 . \mathrm{Kg} 6{ }^{1 / 2-1 / 2}$.

Helmut Conrady mined some 5-man EGTs in 2003 specifically to find the longest AUM sequences: he published eight, q.v., HHdbIV\#70230-7, including the longest known, HHdbIV\#70232, q.v., Figure 2c. KRKPN, 8/8/7p/7n/k7/8/2K5/3R4 w:

1.Rd4+ Ka3 2.Kd3 Kb3 3.Rd6 Kb4 4.Kd4 Nf4 5.Rb6+ Ka5 6.Rf6 Ne2+ 7.Kc4 Ng3 8.Rg6 Ne4 9.Kd4 Nd2 10.Rg2 Nf3+ 11.Kc5 Ka6 12.Rg6+ Kb7 13.Kd5 h5 14.Rh6 h4 15.Rf6 Ne1 16.Rf1 Nc2 17.Rb1+ Ka6 18.Rb2 Ne1 19.Ke4 h3 20.Rb1 Nc2 21.Kd3 Na3 22.Ra1 h2 23.Rxa3+1-0.

\section{References}

Beasley, J. (2003). Depth and Beauty: The chess endgame studies of Artur Mandler. Beasley, Harpenden.

Bleicher, E. (2011). http://www.k4it.de/ Sub-7-man chess EGT-query service.

Bleicher, E., Haworth, G.M ${ }^{\mathrm{c}}$ C. and van der Heijden, H.M.J.F. (2010). Data-mining chess databases. ICGA Journal, Vol. 33, No. 4, pp. 212-214.

Conrady H. (2003). Computerschach und Spiele, Vol. 2, No. iv-v.

Haworth, G.M $M^{\mathrm{c}}$ C. (2009). The Scorched Earth algorithm. A presentation to the Chess Endgame Study Circle, Pushkin House, London, UK, 2009-04-03.

Nalimov, E.V., Haworth, G.Mc C. and Heinz, E.A. (2000). Space-Efficient Indexing of Endgame Tables. ICGA Journal, Vol. 23, No. 3, pp. 148-162. ISSN 1389-6911.

van der Heijden, H. (2010). http://www.hhdbiv.nl/. ENDGAME StUdY DATABASE IV.

\footnotetext{
${ }^{3}$ For example, a class of moves quickly seen as TWMs include many where White safely reverses its previous move.

${ }^{4}$ e.g., Q7/8/8/7K/7p/8/7p/6k1 w, position 7w in Aloni Study HHdbIV31073: White and Black are in effect minimaxing moves to KQK rather than to mate or conversion of force. Thus, White plays 7.Qa1+ rather than 7.Qg8+.

${ }^{5}$ As DTM is not the only metric, there will be positions where DTM-suboptimal but DT $x$-optimal moves are best.

${ }^{6}$ With format (HHdbIV study index, AUMS length and start of AUMS), they are: $(49882,43,8),(71205,37,1)$, (71968, 36, 12), (69257, 35, 5), (75809, 34, 1), (75715, 33, 2), (68418, 26, 1), (38012, 25, 1), (62681, 23, 6), ..

${ }^{7}$ (70232, 23, 1), (70230, 20, 1), (75845, 18, 1), (17426, 15, 1), (69287, 15, 1), (72829, 15, 3), (76116, 15, 1), ...
} 